\title{
Las potencialidades de la Teología Empírica para el desarrollo de una teología en diálogo con la experiencia religiosa actual
}

\author{
Catalina Cerda-Planas \\ Pontificia Universidad Católica de Chile \\ cacerdap@uc.cl
}

Resumen: Ante el desafío aún vigente de que la teología integre, de una manera sistemática y metodológicamente fundada, a la experiencia religiosa actual dentro de sus procesos de generación de conocimiento, en el siguiente artículo se propone a la denominada Teología Empírica como una posible respuesta a dicho desafío. Para ello, se presentan sus principales presupuestos y su metodología, tal como lo sistematiza J. van der Ven, uno de sus principales exponentes, para luego comentar los aportes más relevantes de esta propuesta al problema planteado inicialmente.

Palabras claves: Teología Empírica, método teológico, Teología Práctica, experiencia religiosa actual.

Abstract: Faced with the challenge still in force for theology to integrate, in a systematic and methodologically grounded way, the current religious experience within its knowledge generation processes, the following article proposes the so-called Empirical Theology as a possible response to this challenge. To do so, its main pre-assumptions and its methodology are presented as systematized by J. van der Ven, one of its main exponents, followed by an analysis of the most relevant contributions of this proposal to the problem initially raised.

Key words: Empirical Theology, theological method, Practical Theology, current religious experience.

\section{INTRODUCCIÓN}

El año ${ }^{1} 2015$, la Facultad de Teología de la Universidad Católica de Argentina celebraba 100 años de vida, momento en el cual se realizó un congreso internacional dedicado a reflexionar en torno a la recepción del

$1 \quad$ El presente artículo es una síntesis de la tesis presentada para la obtención del grado de Magister en Teología Fundamental el año 2017 en la Pontificia Universidad Católica de Chile. 
Concilio Vaticano II en el continente. En dicho contexto, el Papa Francisco envió un videomensaje a los participantes en el cual recordó la necesidad que tiene la teología de repensar cómo el cristianismo se hace carne hoy, en un permanente diálogo con el contexto que le toca vivir, pues

no se es cristiano de la misma manera en la Argentina de hoy que en la Argentina de hace 100 años. No se es cristiano de la misma manera en la India, en Canadá, que en Roma. Por lo que una de las principales tareas del teólogo es discernir, reflexionar: ¿qué significa ser cristiano hoy "en el aquí y ahora"? 2.

Tal como él mismo lo recuerda, fue esta también una de las principales tareas que el Concilio Vaticano II le encargó a la teología, luego de ser él mismo un ejercicio de diálogo con y reflexión teológica del tiempo particular en que este fue celebrado hace ya más de 50 años. La intuición fundamental es que la historia ya no puede ser considerada como un terreno neutro al que la fe se le aplicaría como un factor meramente externo. Por tanto, como sintetiza Berríos, "no se trata ya de "bautizar» al mundo para hacerlo luego digno de ese bautismo, sino de descubrir en él mismo los impulsos, más o menos ocultos, del reinado de Dios que interpela" ${ }^{\prime}$.

Se advierte aquí que "la evolución de la vida social", es decir, la historia, no es presentada como destinatario o beneficiaria de la acción eclesial. Por el contrario, emerge claramente, la sociedad percibida como "lugar teológico", como instancia o fuente a partir de la cual se produce un conocimiento teológico, una profundización en la verdad manifestada en el Evangelio de Jesús. [...] se advierte aquí una nueva manera de pensar que se hace cargo del carácter histórico y social del pensamiento mismo y que, de este modo, llega a dar una nueva definición de la relaciones entre Iglesia y sociedad ${ }^{4}$.

Por ello, la teología posterior deberá profundizar en este locus theologicus

2 Papa Francisco, "Videomensaje del Santo Padre al Congreso internacional de teología organizado por la Pontificia Universidad Católica Argentina (Buenos Aires, 1-3 de septiembre de 2015) | Francisco", 2015, acceso el 22 de marzo de 2019, https://w2.vatican.va/content/ francesco/es/messages/pont-messages/2015/documents/papa-francesco_20150903_videomessaggio-teologia-buenos-aires.html.

3 F. BerRíos, "Antecedentes y recepción de Gaudium et Spes en Latinoamérica. Una mirada desde Chile", en Teología de los signos de los tiempos latinoamericanos. Horizontes, criterios y métodos (Ediciones Universidad Alberto Hurtado, Santiago 2013) 27.

4 C. Schickendantz, "Una Elipse Con Dos Focos. Hacia Un Nuevo Método Teológico a Partir de Gaudium et Spes," en Teología de Los Signos de Los Tiempos Latinoamericanos: Horizontes, Criterios y Métodos (Ediciones Universidad Alberto Hurtado, Santiago 2013) 75. 
-la historia- que vuelve a ser reconocido como tal, rechazando cualquier paralelismo entre la realidad de Dios y la realidad del tiempo, y reconociendo su íntima unión aunque sin confusión. "Acudir a estos «lugares» no es optativo, si no constitutivo para la teología"s. Por ello, el Papa vuelve a recordar la tarea aún vigente - $\mathrm{y}$ a ratos pendiente- de que nuestra labor teológica en América Latina sea desarrollada en un profundo diálogo con el tiempo presente, con la fe vivida, con el acontecer histórico actual donde Dios hoy se sigue manifestando:

Las preguntas de nuestro pueblo, sus angustias, sus peleas, sus sueños, sus luchas, sus preocupaciones poseen valor hermenéutico que no podemos ignorar si queremos tomar en serio el principio de encarnación. Sus preguntas nos ayudan a preguntarnos, sus cuestionamientos nos cuestionan. Todo esto nos ayuda a profundizar en el misterio de la Palabra de Dios, Palabra que exige y pide dialogar, entrar en comunicación. De ahí que no podemos ignorar a nuestra gente a la hora de realizar teología. Nuestro Dios ha elegido este camino. Él se ha encarnado en este mundo, atravesado por conflictos, injusticias, violencias; atravesado por esperanzas y sueños. Por lo que, no nos queda otro lugar para buscarlo que este mundo concreto, esta Argentina concreta, en sus calles, en sus barrios, en su gente. Ahí Él ya está salvando .

Evidentemente, la teología ha dado pasos importantes en este sentido, y especialmente en nuestro continente. Las denominadas teologías de la historia o de los signos de los tiempos, desarrolladas con fuerza en Europa y también en América Latina, han sido un esfuerzo concreto por asumir este desafío y continuar lo iniciado por el Concilio en esta línea. Con todo, autores como Noemi han insistido en la vigencia de la pregunta metodológica que surge al alero de esta nueva perspectiva teológico-fundamental. Junto con definir los posibles criterios, es necesario también avanzar en reflexiones

5 C. Schickendantz, "Una Elipse Con Dos Focos...”, 76.

6 Papa Francisco, "Videomensaje del Santo Padre...". 
estrictamente epistemológicas respecto de la teología como inteligencia de la fe y el método más apropiado para la reflexión teológica del tiempo actual:

¿Cómo puede la teología, cómo pueden los teólogos hablar responsablemente del mundo donde viven personas concretas como un lugar en el que son visibles los signos de Dios Creador, Redentor y Fin Último, y cómo desde ahí se puede plantear a Dios y la historia de Dios con la humanidad? [...]. Se trataría de operativizar epistemológicamente un desafío que la Iglesia ha reconocido como verificación de su propia proexistencia para con el mundo? .

Así, el avance que ha significado para la teología del siglo XX volver a reconocer el carácter constitutivamente histórico de la revelación y, por tanto, la cualidad teológica de la historia - de hoy y de todo tiempo-, requiere su correlato en una reflexión propiamente metodológica. ¿Cómo puede la teología incorporar, dentro de su proceso de inteligencia de la fe, la historia actual? ¿Cuáles son las mediaciones disciplinares más adecuadas para el logro de dicho cometido? ¿Cómo puede la teología velar porque el estudio de dicho locus -la historia- mantenga su carácter propiamente teológico?

Motivada por estas preguntas en mi propia labor teológica, llegué a conocer -y a adoptar en mi trabajo- la denominada Teología Empírica, una propuesta metódica que busca reflexionar, de manera sistemática y metodológicamente fundada, el tiempo presente desde una perspectiva teológica. En lo que sigue, presento una muy apretada síntesis del mencionado método para, en un tercer momento, discutir cómo este responde a las preguntas hasta aquí planteadas.

\section{La TeOlogía Empírica: PResupuestos y PROPUeSTa MetOdológica}

\section{1. Antecedentes}

La propuesta que a continuación presento tuvo su origen en Holanda, particularmente en Nijmegen. En 1964, se establecen en la Universidad Católica de Radboud tres nuevas cátedras que marcarán la historia de la Teología Empírica en dicho lugar. Una primera cátedra de Teología Pastoral en la Facultad de Teología y dos cátedras en la de Ciencias Sociales: Sociología y Psico-

7 J. Noemi, "En La Búsqueda de Una Teología de Los «Signos de Los Tiempos»" en Signos de Estos Tiempos. Interpretación Teológica de Nuestra Época (Ediciones Universidad Alberto Hurtado, Santiago 2008) 86-87. 
logía de la Religión. Cuatro años más tarde, se agregaría una cátedra de Educación Religiosa. Cada una de ellas contaba con un equipo y fueron creadas con la intención de "desarrollar una propuesta multidisciplinaria de enseñanza e investigación en Teología Pastoral". Para lograrlo, se propuso abordar en conjunto problemas pastorales y eclesiales pero desde diferentes perspectivas. Así, los fundadores de esta nueva propuesta esperaban desarrollar una Teología Pastoral con un carácter distintivo por su aproximación empírica.

A la base de esta nueva propuesta estaba el Handbuch der Pastoraltheologie, editado por Karl Rahner, en el cual se proponía "el llamado «modelo de dos fases» en el cual una descripción y análisis de algún tema pastoral desde las ciencias sociales era sucedida por una reflexión teológica"9. Con todo, este modelo de trabajo pronto mostró importantes límites y desafió a pensarse nuevas formas de colaboración interdisciplinar en el ámbito de la Teología Pastoral.

Diez años después, el Departamento de Teología Pastoral de Nijmegen vive un vuelco, pasando desde un acercamiento multidisciplinar a intentar el desarrollo de una perspectiva intradisciplinar: a diferencia del modelo hasta ahora implementado, donde cada una de las disciplinas convocadas realiza su investigación desde sus propios supuestos teóricos y metodológicos, que luego son puestos en común en una suerte de yuxtaposición disciplinar, la propuesta ahora es que sean los mismos teólogos pastorales quienes incorporan dentro de sus competencias y su quehacer teológico, las herramientas y técnicas de las ciencias empíricas, en vistas a lograr una mejor comprensión de sus materias de estudio. "En los años sucesivos, a este acercamiento intradisciplinar de la Teología Pastoral de Nijmegen se le comenzó a denominar como "Teología Empírica»"10 por su acercamiento constitutivamente empírico a las materias de estudios".

En su desarrollo reciente, su exponente más representativo ha sido Johannes A. Van der Ven (1940-2019), teólogo práctico holandés, quien fuera profesor de la mencionada Universidad de Radboud en Nijmegen, Holanda. Van der Ven es reconocido por su capacidad de recoger y sistematizar la propuesta en su obra

8 J. van der Ven, Practical Theology: An Empirical Approach (Peeters, Leuven 1998) 1. Todas las traducciones de esta obra son mías.

9 J. van der Ven, Practical Theology..., 2.

10 J. van der Ven, Practical Theology..., 2. 
principal Entwurf einer empirischen Theologie (1990), así como por el aporte, tanto teórico como empírico, que ha hecho a la Teología Práctica contemporánea.

A continuación, entonces presento el método de la Teología Empírica tal como este ha sido sistematizado por Van der Ven. Para ello, primero, expondré una breve síntesis de sus principales supuestos, los que son necesarios para lograr una adecuada comprensión del mismo. Y luego, en un segundo momento, presento la propuesta metodológica propiamente tal.

\section{2. Principales presupuestos de la Teología Empirica de Van der Ven}

Un primer supuesto fundamental de la propuesta dice relación con la comprensión que tiene el autor respecto del objeto de la teología. Van der Ven postula (en la línea de teólogos anteriores como Schillebeeckx) que el objeto directo de la teología no es Dios, como tradicionalmente se afirma, sino de manera más precisa la experiencia que el hombre hace de Dios, a través de la cual el ser humano, y la teología, pueden llegar, de manera indirecta, a decir algo acerca de $\mathrm{E}^{11}$. Ello implica, entonces, la afirmación de que Dios es el objeto directo de la fe y el objeto indirecto de la teología. Por ello, para Van der Ven, la labor teológica está constitutivamente mediada por la experiencia humana. En este sentido, el autor recuerda que toda teología tiene como objeto directo de estudio la experiencia religiosa del ser humano, en sus diversas manifestaciones: escritas, celebradas, reflexionadas, vividas, etc. Cada una de ellas según su particularidad es estudiada de manera específica por las diferentes disciplinas teológicas (Teología Bíblica, Teología Sistemática, Teología Práctica).

Ahora bien, dicho lo anterior, para Van der Ven la Teología Práctica tiene una particularidad dentro de este común objeto de toda teología. Para el autor holandés, esta disciplina teológica pone su foco de estudio en el polo presente de la experiencia, tal como él le denomina. Es decir, su interés particular de estudio está puesto en la experiencia actual, siendo así complementaria a otras disciplinas teológicas que ponen su atención en otros momentos de la experiencia religiosa cristiana. Así, el aporte específico de esta rama teológica es el estudio sistemático y metodológicamente fundado de la experiencia religiosa presente. Y ya no solo como lugar de aplicación de principios teológicos generales a casos particulares del hoy, sino como objeto

11 Cf. J. van der Ven, Practical Theology..., 29. 103-104. 
específico de estudio y, por tanto, como fuente de reflexión teológica (por cierto, en un diálogo hermenéutico con otras fuentes).

En línea con lo anterior, un concepto fundamental para su propuesta será el de praxis. Siguiendo a autores como Habermas y Ricoeur, para Van der Ven la praxis es fundamentalmente una acción hermenéutico-comunicativa lo cual "refiere a la interpretación verbal y no verbal de textos escritos y hablados, y su comunicación verbal y no verbal" ${ }^{\prime 2}$. A través de la interpretación el ser humano busca sobre todo "reconstruir, reflexionar sobre y reinterpretar su propia praxis" 13 . En este sentido, para el autor toda praxis humana es fundamentalmente un acto de interpretación-comunicación entre sujetos o comunidades que buscan establecer y desarrollar el entendimiento mutuo, con la esperanza de poder alcanzar el consenso ${ }^{14}$. Ello supone siempre el encuentro entre dos contextos (o tiempos) diferentes que se ponen en relación. Por ello, la interpretación puede ser comprendida como una acción creativa, pues genera un nuevo texto al incluir el contexto del interlocutor o lector, ajeno, en principio, al texto original.

Por ello, en la fe viva de la Iglesia a lo largo de su historia, el encuentro entre textos del pasado con textos (escritos o vividos) del presente, no es un mero trasvasije de un mensaje fijo del pasado al tiempo presente. Implica una reinterpretación del texto a la luz de la experiencia presente, la que le da un nuevo sentido, integrándose pasado y presente. En consecuencia, el autor recuerda que la relación hermenéutica entre pasado y presente no es simple ni unidireccional:

Este presente no es una situación "vacía de teología" que sólo necesita ser espolvoreada, por decirlo de alguna manera, con el texto del pasado, que entonces serviría como fuente. Lo que hay son dos fuentes que considerar: por un lado, el texto religioso del pasado, que como mucho es el reflejo de las creencias de las personas del pasado, y, por otro lado, la situación del presente, en la cual la fe de personas contemporáneas encuentra su expresión en formas verbales y no verbales contemporáneas. La distancia temporal entre el pasado y el presente existencial es superada por una real comunicación [...]. Esto significa que no existe algo así como el sentido definitivo de un texto del pasado, y ningún sentido puede ser directamente

12 J. van der Ven, Practical Theology..., 41.

13 J. van der Ven, Practical Theology..., 46.

14 El autor aborda in extenso la cuestión de la comunicación al interior de la acción hermenéutico-comunicativa, su orientación al logro del consenso, los criterios para alcanzarlo y las dificultades que surgen en dicho proceso. Cf. J. van Der Ven, Practical Theology..., 49-59. 
"aplicado" al presente. El sentido del texto para el presente sólo puede ser descubierto a partir de lo que se ha llamado "comunicación intergenerativa". En ella, pasado y presente forman un locus theologicus ${ }^{15}$.

La comunicación intergenerativa, mencionada por el autor en la cita anterior, apunta a las múltiples interrelaciones que configuran un proceso hermenéutico: por un lado, el texto se relaciona constitutivamente con el contexto que le dio origen; por su parte, el texto (o persona) actual se relaciona también con su contexto presente. En el proceso hermenéutico ambos se encuentran, interrelacionándose, es decir, se produce un diálogo de relaciones, en un puente interpretativo multidireccional. Por ello, no puede esperarse que la interpretación sea un ejercicio unívoco. Esta es constitutivamente plural.

Un segundo concepto fundamental en la propuesta de Van der Ven es, por cierto, la empeiría. El trasfondo filosófico de la modernidad y del surgimiento de la Teología Empírica estuvo marcado por las corrientes empiristas y pragmatistas desarrolladas por connotados filósofos norteamericanos, como William James y John Dewey. Van der Ven recuerda que, como representante del empirismo, para Dewey ${ }^{16}$ "la experiencia implica un contacto directo, no mediado y "fresco» con los fenómenos de la naturaleza, la sociedad y el ser humano [...]. Las ideas, teorías e hipótesis científicas deben ser verificadas a través de la observación empírica antes de que sean aceptadas como verdaderas y correctas" ${ }^{\prime 1}$. Por su parte, el pragmatismo enfatiza que el conocimiento y el pensamiento deben ser comprendidos como reglas para la acción, focalizando la atención en la dimensión pragmática de los conceptos y sus consecuencias para la acción. Ello tanto desde una perspectiva del método científico como desde una teoría del conocimiento propiamente $\operatorname{tal}^{18}$.

Con todo, el empirismo norteamericano, del cual bebe más directamente la Teología Empírica, rápidamente comprendió que la experiencia es mucho más que algunos datos sensibles de la realidad ${ }^{19}$, abriendo la puerta

15 J. van der Ven, Practical Theology..., 47. Las cursivas son mías.

16 Van der Ven refiere a su artículo "An Empirical Survey of Empiricisms”, en Studies in the History of Ideas, Vol. III (S. 3-22). (Columbia University Press, New York 1935).

17 J. van der Ven, Practical Theology..., 6.

18 Cf. J. van der Ven, Practical Theology..., 12.

19 Cf. J. van der Ven, Practical Theology..., 81. 105. 116-117. 
para integrar, dentro de ella, la experiencia religiosa. Siguiendo esta línea del empirismo, para Van der Ven la empeiría no es únicamente el conocimiento que el ser humano puede adquirir a través de sus sentidos. Para él, la riqueza y complejidad del obrar humano y su interrelación con el medio -ya sea físico, interpersonal o socio-cultural- configuran lo que él entiende por experiencia. Lo anterior posiciona a este concepto dentro del marco de la teoría de la acción humana, y más específicamente, de la inter-acción: el ser humano se interrelaciona con el medio físico, psíquico y social en una relación dialéctica de transformación o de prevención del cambio.

En términos epistemológicos, Van der Ven integra en su propuesta las premisas empiristas y pragmatistas moderadas mencionadas anteriormente, afirmando la necesidad de incorporar la experiencia como parte de los procesos de generación de conocimiento. Mediante la observación empírica, las hipótesis, conjeturas o conocimiento general son testeados, pudiendo ser falsados o corroborados ${ }^{20}$. En el primer caso, la falsación, se confirma que la hipótesis testeada no se da en al menos un caso particular y, por tanto, se demuestra que ella no es válida como afirmación universal. Ahora bien, si mediante la observación se logra mostrar que la conjetura se da en los casos particulares observados, la hipótesis es corroborada, pero sigue manteniendo su carácter hipotético. Mediante la observación, esta se hace más fuerte, más robusta, pero nunca pierde su calidad de tal. De acuerdo al autor, sólo así es posible desarrollar conocimiento, en todo ámbito disciplinar, también en el teológico.

Ahora bien, ¿por qué integrar un acercamiento empírico a la teología? Según el autor, la experiencia puede jugar diversos roles al interior de la teología: ella puede insertarse como actitud (la experiencia religiosa sería una inspiración del trabajo del teólogo), como punto de partida (la experiencia como lugar desde dónde surge la pregunta y el tema a investigar) o como método de investigación (el uso directo de herramientas y métodos empíricos). Para Van der Ven lo central de la propuesta es que la experiencia no sólo forme parte de la teología como inspiración u objeto (que, de hecho, ya ocurre y ha sido ampliamente desarrollado), sino también como método ${ }^{21}$. El aporte específico de ello dice relación con colaborar en la comprensión de las experiencias religiosas actuales dentro del complejo proceso interpretativo que

20 Cf. J. van der Ven, Practical Theology..., 115-17. En esto, el autor sigue fundamentalmente a Popper, refiriendo las siguientes obras: Objective knowledge (Oxford, 1983) y The Logic of Scientific Discovery (London, 1986). 
significa la fe como praxis hermenéutico-comunicativa. En el caso de los textos antiguos y la relación con su contexto, estos son estudiados por acercamientos teológicos de carácter histórico y literario. Ahora bien, en el polo de los textos actuales y su contexto, la mediación empírica se hace necesaria como base para lo que se ha dado en denominar la hermenéutica de las experiencias actuales $\left(\right.$ Schillebeeckx $\left.{ }^{22}\right)$.

[La Teología Empírica] Se dirige de manera sistemática y metódica al polo del presente, tal como éste se manifiesta en la cultura de la sociedad en cuestión, en la iglesia de dicha sociedad, y en el trabajo pastoral. Busca "leer" las "inquietudes fundamentales" del presente y comprender su relación dialéctica con la fe cristiana contemporánea [...]. La teología empírica provee a la teología práctica de las técnicas e instrumentos que le permiten analizar, interpretar y evaluar las convicciones, creencias, imágenes y sentimientos religiosos del hombre y de la mujer ${ }^{23}$.

Desde esta perspectiva, el acercamiento empírico-teológico es sumamente relevante, pues mientras la exégesis y la historia ayudan a profundizar en uno de estos polos, a saber, el pasado y la tradición, la Teología Empírica busca comprender el otro polo, la situación actual, el presente. Y aunque es cierto que el polo de la situación actual ha sido ya considerado en la teología, un acercamiento y estudio sistemático y científico del mismo ha sido históricamente olvidado dentro de ella:

La Teología Empírica [...] provee a la Teología Práctica de los métodos y herramientas para describir y explicar lo que ocurre en las vidas actuales de personas actuales; para analizar y evaluar los textos que estas personas usan para guiar su experiencia religiosa; para explorar y validar las interpretaciones de estas experiencias; $y$, finalmente, para investigar las hipótesis formuladas en relación a estas experiencias y los textos y contextos con los cuales están conectadas. Sin los métodos y técnicas empíricos, la Teología Práctica corre el riesgo de generar solamente apuestas gruesas, asociaciones ingenuas, proyecciones subjetivas o especulaciones irreales ${ }^{24}$.

Ahora, ¿cómo integrar dentro de la teología un acercamiento empírico?

22 El autor refiere aquí a la obra Geloofsverstaan. Interpretatie en kritiek Bloemendaal (1972) de dicho autor.

23 J. van der Ven, Practical Theology.., 109.

24 J. van der Ven, Practical Theology..., 20-21. 
De acuerdo al autor, existen cuatro modelos para responder al desafío de relacionar a la teología con la empeiría como método: la monodisciplinariedad, la mutidisciplinariedad, la interdisciplinariedad y la intradisciplinariedad ${ }^{25}$.

En breve, es posible afirmar que el primer modelo -monodisciplinariedad- tiene como supuesto fundamental la comprensión de la Teología Práctica como aplicación de lo desarrollado por otras disciplinas teológicas a situaciones particulares, teniendo como prioridad fundamental la solución de preguntas en situaciones concretas que no pueden ser replicadas. En este modelo predomina una visión deductiva y unidireccional en la comunicación entre teología y praxis.

La multidisciplinariedad, por su parte, tiene como supuesto fundamental la necesidad que tiene la teología de hacer descripción y análisis de las situaciones concretas que se viven en la Iglesia en el contexto de la sociedad actual, relacionando a la teología con datos empíricos. Para ello, este modelo propone que las distintas disciplinas aborden una misma cuestión (objeto de investigación) cada una desde su perspectiva disciplinar, utilizando sus propios supuestos científicos y metodológicos, sus propias teorías y conceptos, sus propios métodos y técnicas particulares. En la Teología Práctica ello ha devenido en el surgimiento de un método en dos fases: una primera fase, dada por la recolección de datos empíricos por parte de las ciencias sociales acerca del trabajo pastoral y eclesial en la sociedad actual; y una segunda fase, que reflexiona los datos desde un punto de vista teológico, insertándolos dentro de un marco de referencia, teorías y punto de vista normativo dado por la teología.

La diferencia del tercer modelo -interdisciplinariedad- respecto del anterior, y su novedad de acuerdo al autor, es que tensiona hacia la interacción, la reciprocidad y la relación cooperativa entre teología y ciencias sociales, superando así el carácter secuencial de la relación entre ellas que se suele dar en un modelo multidisciplinar. Sin embargo, de acuerdo a Van der Ven, el diálogo interdisciplinar entre teólogos prácticos y cientistas sociales es siempre dispar, dado que los primeros dependen, para su ejercicio, de los segundos, y no viceversa. Además, un modelo como este supone que ambos especialistas tengan un conocimiento suficiente de la otra disciplina como para poder identificar y analizar críticamente los supuestos teóricos y metodológicos que entran en juego en el diálogo interdisciplinario. 
Por su parte,

El modelo intradisciplinar requiere que la teología misma sea empírica, es decir, que expanda su rango tradicional de instrumentos, que consisten en métodos y técnicas literarias, históricas y sistemáticas, en la dirección de una metodología empírica. Esta expansión puede ser descrita por el término intradisciplinariedad que, en un sentido epistemológico general, refiere a la adopción de conceptos, métodos y técnicas de una ciencia por parte de otra, y a la integración de dichos elementos en aquella otra ciencia ${ }^{26}$.

De acuerdo al autor, este modelo de ejercicio intradisciplinar es fundamental para el logro del cometido teológico. De hecho, no sólo en el caso de la Teología Práctica, sino también en otras subdisciplinas: es imposible comprender la teología de santo Tomás de Aquino sin la filosofía aristotélica, o la teología desarrollada en Tübingen en la primera mitad del siglo xIx sin el idealismo filosófico, o la teología de Rahner sin Kant y Hegel. Y ello no sólo en relación a la filosofía; también la integración, dentro de la Teología Bíblica, de la crítica literaria o del análisis estructural y semiótico son muestra de aquello, entre otros tantos posibles ejemplos. Por ello, Van der Ven concluye que el ejercicio de la Teología Práctica y la incorporación del acercamiento empírico dentro de ella requiere y supone un modelo intradisciplinar.

\section{3. Propuesta metodológica de la Teología Empírica}

Ahora bien, ¿̨ómo se materializa todo lo dicho hasta aquí en una propuesta metodológica adecuada para el estudio teológico de la experiencia humana de Dios en el polo presente? Por razones de extensión, a continuación se presenta una síntesis muy apretada de las cinco fases que conforman el método, el cual se sugiere consultar directamente en la obra original para su mejor comprensión ${ }^{27}$.

2. 3. 1. Elaboración del problema y objetivo teologicos (development of the theological problem and goal)

Un primer paso fundamental en el desarrollo de una investigación empírico-teológica es la definición del problema a investigar, es decir, la explicitación de cuál es la temática general que se abordará a través de ella.

26 J. van der Ven, Practical Theology..., 101.

27 Cf. J. van der Ven, Practical Theology..., 119-156. 
Evidentemente, en este caso, la cuestión a estudiar debe ser teológica. Por tanto, es la fe, como objeto directo de estudio, y Dios como objeto indirecto, los que otorgan la naturaleza teológica a la investigación empírica.

Habiendo identificado el tema general de estudio, es también necesario explicitar el sentido u objetivo último de la investigación. Dentro de la propuesta de la Teología Empírica, la fe no es únicamente el objeto directo de investigación, sino también su sentido último ya que la investigación empírica dentro de la Teología Práctica se orienta a la transformación de la praxis hermenéutico-comunicativa de la fe.

\section{3. 2. Inducción teológica (theological induction)}

Esta segunda fase, denominada por el autor como inducción es definida como:

La observación, dirigida por la reflexión, de los fenómenos en la realidad empírica. Esta incluye el descubrimiento y nombramiento de las clases o tipos de fenómenos, el descubrimiento de patrones en los fenómenos, y el develamiento de relaciones comparativas, correlativas y causales entre los fenómenos ${ }^{28}$.

Ahora bien, esta fase está conformada por cuatro elementos: la percepción teológica, la reflexión teológica, la formulación de la pregunta teológica y el diseño de la investigación empírico-teológica.

La primera de ellas apunta a una observación más detallada e intencionada de la temática a investigar tal como ella se da en la realidad empírica. Generalmente se vincula esta sub-etapa a la actividad de un observador más o menos objetivo o neutro que, con la ayuda de instrumentos adecuados, registra observaciones de la realidad. Sin embargo, Van der Ven enfatiza que la percepción se relaciona permanente y dialécticamente con la reflexión, dado que no existe la recolección de datos (sistemática o no) libre de toda teoría; los hechos siempre son percibidos dentro y desde algún tipo de marco de referencia.

Para ello, el autor plantea que se deben cumplir dos condiciones: el investigador debe lograr un conocimiento sinóptico de la literatura teórica más relevante en relación a la temática estudiada. Ello asegura que la re- 
flexión acerca de la percepción sea guiada por la teoría y ayuda a tener una mirada más aguda y profunda. Pero, además, el investigador debe conocer y comprender la literatura empírica pertinente, tanto dentro de la misma teología como de las ciencias sociales. Ello puede entregar nuevas luces a los resultados de la percepción, ya sea confirmándolos, ayudando a verlos desde una nueva perspectiva o complementándolos.

Finalmente, el autor explicita que los insumos obtenidos de la revisión bibliográfica teórica y empírica deben ser extrapolados y aplicados a los descubrimientos de la propia investigación, lo que enriquece, delimita y profundiza la mirada de los datos. Pero a su vez, los datos permiten mirar la literatura de manera crítica, dando criterios de delimitación o discriminación de la misma. Así, la reflexión se relaciona dialécticamente con la percepción en una relación en ambos sentidos.

Fruto de lo anterior, se podrá definir la pregunta teológica específica que orientará la investigación. Ello permite delimitar con mayor precisión lo que se quiere estudiar. No todos los aspectos de un problema requieren o pueden ser investigados. La pregunta se establece en términos precisos, focalizando en solo algunos de los aspectos incluidos dentro del tema de estudio definido inicialmente, privilegiando aquellos que son factibles de ser estudiados teológico-empíricamente.

A la luz de lo anterior, en el último momento de la fase de inducción corresponde elaborar el diseño de la investigación que puede ser comprendido como una "explicitación de la pregunta empírico-teológica" ${ }^{29}$ antes definida. Ello supone tomar opciones en relación al universo y la muestra de estudio, los datos que se requerirá recolectar, los plazos y los métodos operacionales y analíticos que se utilizarán.

\section{3. 3. Deducción teológica (theological deduction)}

Tal como se vio en la descripción de la fase anterior, la inducción es un primer encuentro con los datos empíricos que permite identificar algunas regularidades que, de la mano de la reflexión, harán posible levantar la pregunta o hipótesis de investigación. Hecho aquello, es necesario, en este tercer momento, conceptualizar teórica y operativamente los principales supuestos o elementos involucrados en la investigación recientemente definida:

29 J. van der Ven, Practical Theology..., 127. 
Antes de que las conjeturas e hipótesis sean aplicadas concretamente, deben ser necesariamente formuladas en la forma de conocimiento general. Estas conjeturas e hipótesis generales son formadas conectándolas con las teorías establecidas, las que son adaptadas, modificadas y reestructuradas. En el ámbito de la investigación empírico-teológica, las teorías globales relevantes son, por supuesto, primariamente las teológicas [...]. Estos ejercicios en teología teórica son necesarios en vistas a situar los conceptos obtenidos por inducción en un marco de referencia más amplio, más generalmente reconocido, para distinguirlos más clara y rigurosamente uno de otro, así como para reconocer formas mixtas. La formación de teoría teológica es necesaria para poder analizar, por abstracción y diferenciación, las observaciones obtenidas inductivamente ${ }^{30}$.

Para ello, la fase de la deducción "requiere de la formación de teorías sólidas y conceptualización de los términos usados en la pregunta de investigación" ${ }^{31}$.

La elaboración del modelo conceptual tiene una importancia nuclear para la posterior operacionalización de la investigación. Para lograrlo, se deben definir tres elementos: los conceptos o variables (características), las relaciones entre ellos y las unidades de investigación (individuos, grupos, instituciones, sistemas). La elaboración del modelo teológico-conceptual supone la explicitación de las variables, de los conceptos que las definen y el tipo de relación que se buscará estudiar. A partir de aquello será entonces posible elaborar hipótesis, las que no son arbitrarias, sino que se basan en teorías y estudios empíricos revisados en las fases anteriores y que hacen que la conjetura sea plausible. Tal como se ha dicho anteriormente, si estas hipótesis no son falseadas por el estudio empírico-teológico, ellas serán fortalecidas como tal aunque no se pueda afirmar que hayan sido probadas.

Finalmente, para que los conceptos obtenidos en el proceso de elaboración de la teoría teológica y su definición en variables puedan ser aplicados a la realidad empírica se requiere un paso intermedio: la operacionalización. En ella, los conceptos son redefinidos de tal manera que "incluyan la descripción de las operaciones por las cuales uno pueda determinar si el fenómeno al cual los conceptos se refieren ocurre en la realidad empírica, así como, de ser

30 J. van der Ven, Practical Theology.., 116.

31 J. van der Ven, Practical Theology..., 128. 
posible, la cualidad de dicha ocurrencia" ${ }^{32}$. La operacionalización se realiza, concretamente, definiendo con claridad el tipo de preguntas con las cuales se buscará investigar las relaciones entre variables (abiertas, precodificadas o una combinación de ambas). Para ello, se requiere de la definición lo más precisa posible de los conceptos a utilizar.

Una vez terminado el proceso de elaboración del instrumento, es necesario definir el grupo específico de personas a las cuales se quisiera estudiar. Ello incluye dos aspectos diferentes: cuál es la población que se quiere estudiar (universo) y la muestra para realizarlo (población muestral). Por cierto, ambas decisiones dependerán del objetivo del estudio y los criterios para su definición dependerá también de la metodología específica de levantamiento de datos que se haya elegido: cuantitativa, cualitativa o mixta.

\section{3. 4. Testeo empirico-teológico (empirical-theological testing)}

La formulación del modelo teológico-conceptual y su operacionalización son sucedidos por su testeo con la realidad empírica, poniendo a prueba su veracidad. Evidentemente, el testeo de las hipótesis o de las preguntas de investigación que surgieron de las fases previas debe ser realizado en un material empírico distinto al que se utilizó en la fase de inducción, para evitar una confirmación espuria de las mismas. Fruto de esta cuarta fase será la falsación o corroboración de aquellas.

El testeo teológico incluye como núcleo central el levantamiento de datos, es decir, a la aplicación de las encuestas, la realización de las entrevistas o la ejecución de la metodología específica de investigación que se haya seleccionado.

Teniendo entonces los datos disponibles es posible pasar a la fase del análisis de los mismos, la cual se estructurará dependiendo de la metodología empírica específica que haya sido escogida. En cualquier caso, lo que se debe lograr en esta fase es la descripción y explicación de los resultados en un primer nivel de análisis de los mismos. Ello, como base fundamental para el ejercicio de análisis teológico en la etapa que sigue. 


\section{3. 5. Evaluación teológica (theological evaluation)}

Habiendo hecho el primer análisis descriptivo de los resultados, en este momento se busca "realizar la interpretación teológica pues los datos obtenidos en la fase analítica precedente no proveen, por sí mismos, una respuesta a la pregunta teológica planteada. Más bien, los resultados requieren ser interpretados"33, puestos en relación con el contexto teológico que se explicita en la pregunta y objetivo teológicos y que fue ampliamente desarrollado a través de los conceptos y teorías que estuvieron a la base de la investigación.

Este paso dentro de la evaluación implica poner los resultados en el contexto de un marco de referencia más amplio: el teológico-hermenéutico. Los datos empíricos solo son significativos cuando son puestos en el contexto hermenéutico de conceptos y teorías teológicas y son evaluados dentro de él. A su vez, las teorías pueden y deben ser enriquecidas por los datos de la realidad que han sido puestos de relieve a través de la investigación empírica:

[El teólogo] debe preguntarse por qué las personas han exhibido estas preferencias, en qué condiciones teológicas este fenómeno puede estar basado, qué significan estas condiciones teológicas y qué lugar deben ocupar en una reflexión teológico-hermenéutica actualizada. Vemos, entonces, dos peligros que deben ser evitados aquí: empirismo teológico, por un lado, y anti-empirismo teológico, por el otro. El proceso reflexivo involucrado aquí es dialéctico: los resultados del estudio empírico ejercen influencia sobre la reflexión teológico-hermenéutica, sin la cual se estaría enfrentando a una dictadura empírica. Al mismo tiempo, sin embargo, una mayor reflexión teológico-hermenéutica estimula una renovada confrontación con la realidad empírica ${ }^{34}$.

Así, y tal como se planteó al abordar los elementos teóricos que componen la propuesta de Van der Ven, la investigación empírica al interior de la teología permite el diálogo hermenéutico entre, por un lado, la situación o experiencia religiosa actual y su contexto, $y$, por otro, la experiencia religiosa pasada y su contexto, y entre ellas, generando un diálogo de relaciones hermenéutico-comunicativo y, en este caso, teológico:

Sólo en el "diálogo de relaciones", en el cual los símbolos del pasado son relacionados con el contexto pasado y los símbolos del presente con

33 J. van der Ven, Practical Theology..., 152.

34 J. van der Ven, Practical Theology..., 153. 
el contexto presente, y luego viceversa, es que el proceso dialéctico del pensamiento, el movimiento entre el antes y el ahora, llega a ocurrir [...]. La teología consiste en una comunicación hermenéutica permanente dirigida al desarrollo del entendimiento en el contexto de la búsqueda del consenso ${ }^{35}$.

Finalmente, Van der Ven menciona la importancia de realizar una mirada crítica al proceso mismo de investigación empírica en cada una de sus fases y sub-fases, a la luz de la experiencia hecha. Por ejemplo, es posible que se descubra que es pertinente modificar el planteamiento del problema y afinar la pregunta de investigación; así mismo, es posible concluir que la conceptualización y, por tanto, el modelo conceptual elaborado, requieren de adaptaciones por mostrar imprecisiones a la hora del testeo; por su parte, la operacionalización es uno de los elementos que debe, sin lugar a dudas, ser evaluado dada la relevancia que juegan los instrumentos de investigación dentro del proceso. Todo lo anterior no implica negar el valor de la investigación realizada, sino que apunta al proceso permanente de desarrollo del conocimiento.

\section{El valor del método de la Teología Empírica para el estudio teoló- GICO DE LA HISTORIA ACTUAL}

Presentados de modo general el método y sus supuestos, en este tercer apartado se intentarán identificar sus principales aportes al problema que ha orientado este artículo, visualizando en qué medida la Teología Empírica logra abordar la cuestión abierta en el primer apartado.

\section{1. Teología y experiencia}

Un primer aspecto fundamental al cual me parece que la propuesta de Van der Ven aporta es al esclarecimiento de la relación entre teología y experiencia. Tal como se vio antes, el autor comprende, de la mano de teólogos como Schillebeeckx y Schoonenberg, que el objeto directo de la teología es siempre la experiencia que el hombre hace de Dios. Y que solo a través de ella el ser humano puede llegar a conocer y decir algo acerca de Él. Así el autor retoma una intuición fundamental de la teología contemporánea, la cual ha vuelto a reconocer el carácter histórico de la revelación y, por tanto, 
que ella está constitutivamente mediada por la experiencia humana ${ }^{36}$. De hecho, incluso fuentes principales de la revelación, como son las Sagradas Escrituras y la Tradición, son expresión de la experiencia creyente de un pueblo, de comunidades o de la Iglesia en su conjunto. Ello permite reconocer que toda teología -no solo la Práctica- tiene como objeto directo de estudio la experiencia religiosa del ser humano, en sus diversas manifestaciones escritas, celebradas, reflexionadas, vividas, etc. ${ }^{37}$.

Evidentemente, ello supone una comprensión amplia del concepto de experiencia el cual, tal como ya se vio, para Van der Ven apunta a la complejidad de la interrelación entre la persona y su medio ya sea físico, interpersonal, social o cultural. Así, el autor supera, de la mano de la epistemología contemporánea, la estrechez con que algunas veces se comprendió la experiencia en los inicios de las corrientes filosóficas empíricas al reducirla únicamente a la dimensión sensible de la percepción humana.

Lo anterior hace posible afirmar una premisa que es fundamental para la Teología Empírica: que teología y experiencia no son ajenas, que no se oponen y que, más aún, ambas están íntimamente ligadas, dado que la segunda es un supuesto sine qua non de la primera. Ello permite superar ciertas suspicacias infundadas que existen hacia la posibilidad de estudiar la experiencia religiosa actual, integrando dentro de la teología un acercamiento empírico, cada vez que se cuestiona su carácter propiamente teológico al argumentar que ello no es hablar de Dios, sino del ser humano. Desde una perspectiva cristiana, Dios y hombre no se oponen ni compiten, sino que Aquel se manifiesta a través de este.

Pero, además, pone en evidencia una de las consecuencias lógicas de la afirmación del carácter histórico de la revelación: que ella solo es accesible a través de la historia y de la experiencia humana, en todos sus momentos y expresiones. Y que, por tanto, la teología solo puede desarrollar su labor de generación de conocimiento acerca de Dios a través del estudio de la expe-

36 Ello no niega ni desconoce, por cierto, el carácter trascendente y gratuito de esta, tal como insistirá Rahner, y como recordará también Van der Ven al abordar este tema. Cf. K. RahneR, Curso Fundamental de La Fe (Herder, Barcelona 2007) 155.

37 Cada una de ellas y según su particularidad es estudiada de manera específica por las diferentes disciplinas teológicas (cf. R. Ganzevoort, "Van Der Ven's Empirical/Practical Theology and the Theological Encyclopaedia", en Hermeneutics And Empirical Research In Practical Theology: The Contribution Of Empirical Theology By Johannes A. Van Der Ven [Brill, 2004] 53-74). 
riencia religiosa (pasada y actual) ${ }^{38}$. En este sentido, propuestas metódicas como estas permiten dar el lugar que le corresponde a la historia como lugar teológico en el diálogo hermenéutico que constituye a la labor teológica. En este sentido, no se espera que la experiencia actual se establezca como norma teológica sin más; ello sería una ingenuidad y un error en la comprensión del desarrollo del pensar teológico. Lo que aquí se busca enfatizar es la necesidad de considerar y no dejar fuera del pensar teológico -como muchas veces ha pasado-a la experiencia humana y religiosa actual como una fuente relevante y necesaria.

\section{2. Acercamiento empirico en teología}

Ahora bien, en el apartado anterior también se vio que para Van der Ven la Teología Práctica tiene una particularidad dentro de este común objeto de toda teología. Para el autor holandés, esta disciplina teológica pone su foco de estudio en el polo presente de la experiencia de la fe, tal como él le denomina. Es decir, su interés particular de estudio está puesto en la experiencia actual, siendo así complementaria a otras disciplinas teológicas que ponen su atención en otros momentos de la experiencia religiosa cristiana. Así, el aporte específico de esta rama teológica es el estudio sistemático y metodológicamente fundado de la experiencia religiosa presente.

Lo anterior ha supuesto una importante consecuencia metodológica para la mencionada disciplina. Van der Ven postula que el logro de dicho cometido específico requiere que la teología integre, dentro de su proceso investigativo, un acercamiento particular a la experiencia: el empírico ${ }^{39}$. Dicho acercamiento empírico dentro de la teología puede, por cierto, materializarse a través de diversas mediaciones disciplinares (por ejemplo, a través del arte, la literatura, las ciencias sociales, entre otras), aunque el teólogo holandés opte por estas últimas.

En este sentido, la riqueza de la propuesta de Van der Ven es que ella

38 En tierras latinoamericanas, Sergio Silva ya hacía eco de esta claridad en un artículo del año 1989: "La teología es un esfuerzo por estudiar sistemáticamente el encuentro entre Dios y el hombre" (S. SiLva, "Ensayo sobre las condiciones actuales del quehacer teológico y sobre su estatuto teórico", en Teología y Vida 30/3 [1989] 155).

39 En este aspecto, se ha logrado ya un consenso bastante amplio dentro de la Teología Pastoral o Práctica, la cual ha tomado conciencia de la necesidad de incorporar metodologías de investigación empírica para un mejor estudio de la experiencia creyente actual o religión vivida (cf. Á. Granados, Identidad y Método de La Teología Pastoral [Edicep, Valencia 2010]). 
desafía a la propia teología a realizar dicho proceso de estudio metódico de la experiencia religiosa actual a través de la incorporación de un acercamiento empírico. Otra opción sería, como en variadas ocasiones y propuestas ocurre, recoger lo que otras ciencias afines, como la psicología religiosa o la sociología de la religión, logran estudiar en torno a ella. Sin embargo, la mera interpretación teológica de datos empíricos recogidos desde otras disciplinas conlleva la yuxtaposición de presupuestos tanto teóricos como metodológicos que hacen que la teología pierda su debido lugar en el proceso de generación de conocimiento en torno a la experiencia religiosa actual ${ }^{40}$. Finalmente, lo que define a una disciplina no es tanto su objeto material, sino la perspectiva desde la cual se estudia dicho objeto.

Por su parte, para lograr una adecuada y fructífera integración del acercamiento empírico dentro de la teología, Van der Ven propone la utilización de un modelo intradisciplinar. Con ello, el autor refiere a la incorporación, en este caso, de procedimientos y técnicas de las ciencias sociales empíricas dentro del proceso de investigación teológica. La riqueza de su propuesta para la Teología Práctica es que son los propios teólogos quienes incorporan dentro de su labor las competencias y los métodos necesarios para el conocimiento cada vez más acabado de la religiosidad actual y sus transformaciones. Así, la propuesta del autor holandés -y una de sus principales novedades, a mi modo de entender- es la realización de una investigación transversalmente empírico-teológica. Ello se materializa, además, en una propuesta metódica minuciosamente desarrollada, en la cual se evidencian los fundamentos espistemológicos y se presentan en detalle las fases y subfases que la componen. Ello le da a la propuesta de Van der Ven una claridad epistemológica y metodológica que es difícil de encontrar en otras propuestas similares.

En el caso de nuestro contexto latinoamericano, además, me parece que una propuesta como la aquí analizada nos ayuda a superar la yuxtaposición disciplinar que ha caracterizado a la teología del continente desde el surgimiento de la Teología de la Liberación, cuyo intento por reflexionar teológicamente la realidad oprimida de América Latina implicó la necesidad de lo que sus principales exponentes llamaron la "mediación socio-analítica" -refiriéndose por ello a los aportes teóricos de las ciencias sociales que le

40 Cf. J. Silva, "Theory and Empirical Research in Liberation Theology", en ET-Studies 7/1 (2016) 159. 
permiten a la teología tener una mirada menos ingenua de la realidad ${ }^{41}-$, la cual siempre fue considerada como un momento "pre-teológico"42. Ello supuso la yuxtaposición de un análisis teológico al diagnóstico empírico sobre el presente ${ }^{43}$, quedando abierto el desafío de incorporar esta mediación no "como algo que ocurre «antes» o «fuera» de la teología, sino como un momento propio e interno" 44 , desafío al cual, me parece, la Teología Empírica aquí presentada aporta de manera directa.

\section{3. Teoría teológica y empeiría}

Ahora bien, tal como ya es posible atisbar, lo anterior tiene como supuesto fundamental, en el pensamiento del autor, una relación dialéctica entre teoría (teológica) y empeiría, en la cual teoría y conocimiento empírico se condicionan y aportan mutuamente. A ello colabora de manera directa la comprensión que el autor tiene acerca de la experiencia humana como inter-acción, es decir, como interrelación del hombre con el medio físico, psíquico y social en una relación recíproca. En ese sentido, para el teólogo holandés la experiencia presente está constitutivamente vinculada tanto a su propio contexto como al pasado que la constituye y le permite llegar a ser, siendo ella misma una forma de praxis hermenéutico-comunicativa.

Ello implica, a nivel epistemológico, que la experiencia actual del ser humano solo puede ser vivida y conocida, incluso empíricamente, desde los supuestos teóricos que orientan (ya sea de forma temática o atemática) toda acción y todo proceso investigativo. Por tanto, la perspectiva interpretativa de los datos empíricos (en este caso, de la experiencia creyente desde el marco de la tradición teológica) no es un agregado ajeno a su comprensión de la experiencia y, por tanto, al método, sino un elemento configurador del mismo. En ese sentido, solo a la luz de la tradición teológica es que la experiencia religiosa actual, recogida a través del acercamiento empírico, puede ser comprendida.

El marco de referencia teórico dentro del cual el investigador recolecta los datos es hermenéutico por naturaleza, dado que el investigador se

41 Cf. J. Silva, "Theory and Empirical Research in Liberation Theology", 155.

42 Cf. C. Boff, Teología de lo politico: sus mediaciones (Sígueme, Salamanca 1980) 19.

43 Cf. J. Noemi, "En la búsqueda de una teología de los «signos de los tiempos»", 84-85. Cf. también S. Sirva, "La Teología de la Liberación”, en Teología y Vida 50/1-2 (2009) 93-116.

44 J. Silva, "Theory and Empirical Research in Liberation Theology", 159. 
sostiene, independiente de si él es consciente o no, en una o más tradiciones culturales y teóricas, las cuales él, una y otra vez, interpreta (y se re-apropia) y cuya relación él no puede hacer desaparecer, dado que, de otro modo, estaría cortando la rama en la cual él mismo está sentado. En otras palabras [...] la investigación empírica está envuelta por la interpretación, siendo precedida y sucedida por ella ${ }^{45}$.

Pero, a su vez, la realidad empírica juega un rol no solo como receptora de los posibles aportes desde la teoría teológica en vistas a la transformación de la misma, sino que aparece también como un lugar desde donde proponer pistas de enriquecimiento o corrección de la teoría teológica. Así, la tradición teológica, para ser fecunda y seguir su constitutivo proceso de desarrollo $^{46}$, requiere entrar en diálogo con las actuales experiencias religiosas y las nuevas interpretaciones de la fe que ellas suponen.

En términos epistemológicos, ello se funda en la hermenéutica de Paul Ricoeur ${ }^{47}$, para quien la relación entre ambos conceptos en las ciencias sociales es dialéctica: la teoría intenta comprender cierta praxis desde una determinada perspectiva, comprensión que debe ser críticamente corregida por la explicación (empírica); la que, a su vez, permite enriquecer o corregir dicha comprensión inicial (teórica), generando una nueva comprensión, lo cual permite reiniciar el ciclo en un proceso acumulativo de conocimiento.

Así, el análisis de los resultados de una investigación teológico-empírica implica poner en contexto dichos resultados con un marco interpretativo mayor, configurado principalmente por elementos de teología sistemática. Ello permite, por un lado, interpretar los datos desde los supuestos teóricos que dieron sustento a la investigación y desde el marco hermenéuticocomunicativo que configura a la praxis. Pero, además, tal como plantea el teólogo holandés, permite levantar insumos críticos o de retroalimentación desde los resultados empíricos a las teorías teológicas que sustentaron el marco conceptual y su operacionalización. Tomar en serio a la experiencia creyente actual como fuente de generación de conocimiento supone para la

45 J. van Der Ven, "God: Search and Re-Search" en Religious Individualization and Christian Religious Semantics (Lit, Münster 2001) 66-67.

46 Cf. B. Lonergan, Método en teología (Sígueme, Salamanca 1988); Y. Congar, La tradición y las tradiciones (Dinor, San Sebastián 1964).

47 Cf. C. Hermans, "When Theology Goes 'Practical'. From Applied to Empirical Theology," en Hermeneutics and Empirical Research in Practical Theology: The Contribution of Empirical Theology by Johannes A. van Der Ven (Brill, Leiden 2004) 24. 
teología mirar críticamente sus supuestos conceptuales a la luz de resultados empíricos. Lo que está en juego aquí, por tanto, es el diálogo fecundo entre teología (especulativa) y acercamiento empírico a la experiencia religiosa de cada tiempo, y las potencialidades que ello supone para el desarrollo del conocimiento teológico.

En este sentido, me parece necesario mencionar que la opción principal del autor por utilizar metodologías empíricas de carácter cuantitativo fundadas en perspectivas más bien positivistas- no ha estado exenta de críticas. Una de ellas ha versado comúnmente sobre el supuesto epistemológico que estaría detrás de dicho tipo de metodologías: la relación sujeto-objeto en el proceso de conocimiento y, por tanto, la objetualización de la experiencia religiosa, constitutivamente compleja y, tal como se ha visto en el apartado anterior, hermenéutico-comunicativa. Pero además porque la utilización de metodologías cuantitativas de investigación empírica pareciera no condecirse del todo con la comprensión del autor en torno a la praxis como acción hermenéutico-comunicativa. Dado que el carácter interpretativo de la existencia humana apunta principalmente a los significados que tiene el ser humano del mundo, de la acción y, en este caso, de Dios, parecería que el modo más adecuado de acceder a ellos es principalmente la "comprensión". Por ello, pareciera que metodologías empíricas de carácter cualitativo pueden ser más coherentes con el objeto específico de estudio y una buena línea a indagar para la teología, caracterizada por una reflexión más bien de corte hermenéutico ${ }^{48}$.

Tal vez sea interesante, en este sentido, experimentar, tal como el mismo autor lo propone en un artículo bastante más reciente ${ }^{49}$, con investigaciones de carácter mixto, es decir, que integran tanto el aporte de metodologías cuantitativas como cualitativas en un único proceso investigativo, lo cual ha sido desarrollado con especial fuerza en los últimos años por las ciencias

48 De hecho, existe ya una línea de desarrollo de la Teología Empírica que ha optado por la utilización de métodos cualitativos de investigación al alero de paradigmas epistemológicos de corte hermenéuticos o fenomenológicos. Cf. por ejemplo, R. Ganzevoort, "Reading by the lines: proposal for a narrative analytical technique in empirical theology", en Journal of Empirical Theology 11/2 (1998) 23-40; H. G. НегмвRоск, "From Data to Theory: Elements of Methodology in Empirical Phenomenological Research in Practical Theology", en International Journal of Practical Theology 9/2 (octubre de 2005) 273-99.

49 Cf. J. van der Ven, “Towards a Comparative Empirical Theology of Mindful Action,” en Hermeneutics And Empirical Research In Practical Theology: The Contribution Of Empirical Theology By Johannes A. Van Der Ven (Brill, 2004) 372-73. 
humanas ${ }^{50}$. Ello permitiría recoger el valor de las metodologías cuantitativas, las que hacen posible estudiar los fenómenos en muestras más amplias de la población e identificar posibles relaciones causales entre las variables, intentando no solo describir sino también explicar dichos fenómenos. Pero a su vez, permitiría reconocer la complejidad del objeto de estudio, constitutivamente hermenéutico, lo que requiere superar o al menos complementar el enfoque popperiano que subyace -a nivel epistemológicoa la propuesta de Van der Ven ${ }^{51}$.

Con todo, me parece que la definición de cuál es la opción metodológica empírica específica más adecuada para ser utilizada en cada caso dependerá de la temática, del conocimiento empírico previo con el que se cuente $\mathrm{y}$, por supuesto, de los objetivos propuestos para cada investigación.

\section{4. Aporte a una nueva comprensión de la Teología Práctica}

Finalmente, es valioso comentar también el aporte que Van der Ven ha hecho a la Teología Práctica desde una perspectiva fundamental, dado que con su propuesta el autor ayuda a consolidar un giro epistemológico que esta disciplina venía viviendo en los últimos años de su desarrollo ${ }^{52}$.

En el inicio de la así llamada Teología Pastoral (siglo XIx), esta se comprendió fundamentalmente en relación al clero y su labor de pastoreo de las comunidades cristianas. En este caso, la pregunta era quién y cómo se debe aplicar a la vida concreta de la Iglesia y de los cristianos, de la manera más eficiente y adecuada, la teología (desarrollada por otras áreas disciplinares de esta, principalmente por la Sistemática). Debido a ello, esta primera orientación se configuró, en general, como una colección de reglas de cómo hacer, de métodos y técnicas para la aplicación de la teología a la pastoral, evidenciando así una perspectiva fundamentalmente deductiva.

50 Cf. J. W. CRESWell, Research Design: Qualitative, Quantitative, and Mixed Methods Approaches, 4th. ed.. (SAGE, Los Angeles, Calif. 2014); J. W. Creswell, Designing and Conducting Mixed Methods Research (SAGE, Los Angeles 2011).

51 Cf. R. Brouwer, "Detecting God in Practices: Theology in an Empirical-Theological Research Project", en Hervormde Teologiese Studies 66/2 (2010) 4; H. G. Негмвrock, "From Data to Theory", 276.

52 Cf. J. van der Ven, Practical Theology..., 35-40; R. Ganzevoort, "Forks in the Road when Tracing the Sacred Practical Theology as Hermeneutics of Lived Religion", Paper presented at the International Academy of Practical Theology, 2009; G. De MoRI, "El carácter práctico de la teología", en Teología y vida, 51/4 (2010) 501-519. 
Una segunda orientación de la Teología Pastoral surge gracias a una ampliación en la comprensión de la labor pastoral y de la Iglesia, reconociendo que el verdadero sujeto de aquella no es únicamente el clero, sino toda la comunidad eclesial. El giro está dado entonces desde pensar cómo el clero puede desarrollar mejor sus funciones a una reflexión sobre la vida de la Iglesia, en perspectiva de una eclesiología del Pueblo de Dios desarrollada por el Concilio Vaticano II. Con todo, la perspectiva de fondo en la comprensión de la labor principal de la Teología Pastoral sigue siendo bastante similar, manteniendo el carácter meramente aplicativo de la disciplina, comprensión muchas veces aún vigente tanto en la acción pastoral de la Iglesia como en la teología.

Con todo, los últimos decenios han marcado un nuevo proceso de ampliación de la Teología Pastoral o Práctica -como se le ha preferido denominar-, excediendo los límites de la acción eclesial y localizándose ahora dentro de las coordenadas dadas entre la sociedad, el cristianismo y la Iglesia. Para esta nueva orientación, dentro de la cual se incluye una propuesta como la de Van der Ven, el desafío de la Teología Práctica es reflexionar la experiencia religiosa al interior de la Iglesia y fuera de ella, así como la relación entre los fenómenos religiosos y no religiosos en la sociedad. En el fondo, se busca reconocer que la labor pastoral, incluso dentro de un marco de referencia de acción eclesial, se da en una sociedad con características particulares, en la cual el cristianismo existe y requiere de un adecuado desarrollo. "La Teología Práctica [...] debe dedicarse a una reflexión crítica sobre y a un estudio empírico acerca de las condiciones bajo las cuales la búsqueda de un sentido trascendente ocurre" ${ }^{53}$.

En este sentido, es posible afirmar que el autor, a través de su obra y de la propuesta metódica que él sistematiza, ayuda a posicionar a la Teología Práctica en el contexto de diálogo con otras disciplinas teológicas no solo como lugar de aplicación sino como fuente de generación de conocimiento teológico. Evidentemente, para que ello sea realmente así, se requiere que la teología en su conjunto se abra a incorporar el polo presente de la experiencia religiosa en su labor teológica, poniéndolo en diálogo con otras fuentes como las Sagradas Escrituras o la tradición. Todo lo anterior, en vistas a poder identificar los aportes que la experiencia religiosa actual puede hacer al desarrollo de la teología en general, intentando superar la distancia que a ratos se ha establecido entre teología y vida ${ }^{54}$.

53 J. van der Ven, Practical Theology..., 39.

54 Cf. H. G. Heimвrocк, "Practical Theology as Empirical Theology," en International Journal of 
De ser así, la Teología Práctica podría transformarse en un insumo relevante para la labor de otras disciplinas teológicas, en tanto en cuanto ellas quieran incorporar, dentro de su reflexión, la experiencia creyente actual. Ello permitiría a la teología en general abrirse al aporte que la actual experiencia religiosa tiene para hacer al desarrollo de la teología como inteligencia de la fe. Fe que no solo ha acontecido en el pasado, sino que sigue experimentándose y desarrollándose en los tiempos contemporáneos y, por tanto, su comprensión requiere de un diálogo hermenéutico entre pasado y presente.

Evidentemente, ello no significa que el teólogo sistemático deba desarrollar por sí mismo las competencias y conocimientos para la realización de una investigación teológico-empírica (así como tampoco lo hace respecto de la exégesis bíblica, por poner otro caso). Pero sí supone un trabajo colaborativo entre disciplinas teológicas. Si realmente la teología reconoce a la experiencia creyente actual como un lugar de reflexión teológica, deberá estar atenta a los resultados que teólogos prácticos puedan descubrir desde su campo específico de estudio. Y más aún, deberá potenciar el desarrollo, a través de un trabajo colaborativo, de investigaciones teológico-empíricas en torno a temáticas como podrían ser la cristología, la eclesiología, la antropología teológica, por mencionar solo algunas.

El rol de la investigación empírica de y dentro de la teología es dialéctico. Por un lado, ofrece a la teología información e insumos empíricos acerca de los procesos experienciales, cognitivos, afectivos, conativos, éticos, rituales y comunitarios que las personas viven dentro de su relación con Dios [...]. Esta información es indispensable dado que la teología puede ser definida, en términos generales, como la reflexión académica acerca de la fe, y más específicamente, como la reflexión académica acerca de la fe actual. En este sentido, la investigación empírica se configura como una condición necesaria para la teología. Pero, por otro lado, es una condición insuficiente, dado que junto a la información e insumos empíricos acerca de la fe de las personas, la teología necesita de información e insumos literarios, históricos y sistemáticos, los que todos juntos conforman el material desde el cual la reflexión teológica comienza y se desarrolla crítica y constructivamente ${ }^{55}$.

Practical Theology 14/2 (2011) 155.

55 J. van der Ven, "God: Search and Re-Search", 67. 
Este aporte del autor a la comprensión de la Teología Práctica como disciplina, me parece especialmente valioso para nuestro contexto latinoamericano, dado que le ayuda a evidenciar el estatuto epistemológico que corresponde a la disciplina ya no como una ciencia aplicativa de los resultados de otras disciplinas teológicas, sino como un área de la teología que estudia una fuente diferente y complementaria a aquellas estudiadas por otras disciplinas teológicas. Esta perspectiva -aún en ciernes en nuestro continenteme parece es un anhelo que está latente en los actuales desarrollos teológico-pastorales en América Latina ${ }^{56}$, los que, sin embargo, aún requieren de precisiones epistemológicas y metodológicas que creo la Teología Empírica ayuda a esclarecer.

Para finalizar y a modo de conclusión, creo que la propuesta de Teología Empírica que el autor estudiado ofrece ayuda a reconocer el rol y el lugar que tiene la praxis en la generación del conocimiento teológico, proponiendo, además, un método específico para ello. Un método que, tal como se ha visto, permite hacer un estudio epistemológicamente fundado de la experiencia religiosa actual, del modo más apropiado para ello -el empírico-, manteniendo la perspectiva disciplinar teológica, en vistas a generar un diálogo fecundo entre teoría teológica y experiencia religiosa actual. Por ello, creo que es posible afirmar que la Teología Empírica se constituye como un aporte metodológico pertinente y valioso a la necesidad que tiene la teología de incorporar, dentro de su labor de inteligencia de la fe, a la experiencia religiosa presente, respondiendo así al reconocimiento del valor teológico de la historia y al desafío planteado por el Concilio Vaticano II a la teología.

56 Me refiero a los avances que ya existen en relación a estudios empíricos en el contexto de la teología, desarrollados especialmente en Argentina, pero también en otras latitudes como México y Colombia. Solo a modo de ejemplo, es posible mencionar las múltiples publicaciones de Virginia Azcuy o el estudio latinoamericano compendiado en M. Eckholt y S. Silber, eds., Vivir la fe en la ciudad hoy: las grandes ciudades latinoamericanas y los actuales procesos de transformación social, cultural y religiosa (Eds. Paulinas, Mexico, D. F. 2014). 